\title{
One-Stage Anterolateral Debridement, Bone Grafting, and Internal Fixation for Treating Lumbosacral Tuberculosis
}

\author{
Tao Zhang ${ }^{1 *}$, Lihua Ma ${ }^{2 *}, \mathrm{Xu} \mathrm{Lan}{ }^{1}$, Ping Zhen ${ }^{1}$, Shiyong Wang ${ }^{1}$, Zhilin $\mathrm{Li}^{1}$ \\ ${ }^{1}$ Department of Spine Surgery, Lanzhou General Hospital, Lanzhou Command of Chinese PLA, Lanzhou, China \\ ${ }^{2}$ Endocrinology Department, The First Hospital of Lanzhou University, Lanzhou, China
}

Study Design: Retrospective case series.

Purpose: To investigate the clinical efficacy and feasibility of one-stage anterolateral debridement, bone grafting, and internal fixation for treating lumbosacral tuberculosis.

Overview of Literature: There has been no consensus regarding the optimal means of treating lumbosacral tuberculosis. The onestage anterolateral extraperitoneal approach for radical debridement, bone grafting, and internal fixation for treating lumbosacral tuberculosis is rare in literature.

Methods: Twenty-one patients with lumbosacral tuberculosis were retrospectively analyzed. All patients underwent the surgery of anterolateral debridement after regularly antituberculous drugs therapy. We evaluated the erythrocyte sedimentation rate, C-reactive protein, radiography, computed tomography, magnetic resonance imaging, visual analogue score, and Oswestry disability index before and after surgery.

Results: All patients completed a follow-up survey 9-48 months after surgery. All patients' wounds healed well without chronic infection or sinus formation, and all patients with low-back pain reported relief after surgery. All cases had no tuberculosis recurrence. Solid bony fusion was achieved within 6-12 months. At final follow-up, evaluated the erythrocyte sedimentation rate decreased from $38.1 \pm 12.5$ to $11.3 \pm 7.1 \mathrm{~mm} / \mathrm{hr}$, C-reactive protein decreased from $6.2 \pm 4.2$ to $1.6 \pm 1.3 \mathrm{mg} / \mathrm{dL}$, the visual analog scale score decreased from $4.6 \pm 1.1$ to $1.4 \pm 1.0$, the Oswestry disability index score decreased from $50.2 \% \pm 11.9 \%$ to $13.0 \% \pm 6.6 \%$, and the lumbosacral angle increased from $20.0^{\circ} \pm 4.8^{\circ}$ to $29.0^{\circ} \pm 3.9^{\circ}(p<0.05)$.

Conclusions: One-stage anterolateral debridement, bone grafting, and internal instrument fixation for treating lumbosacral tuberculosis is safe and effective.

Keywords: Lumbosacral tuberculosis; Tuberculous spondylitis; Anterolateral debridement; Extraperitoneal debridement; Internal fixation

\section{Introduction}

Spinal tuberculosis $(\mathrm{TB})$ is one of the most common forms of extrapulmonary $\mathrm{TB}$, accounting for approximately $50 \%$ cases of musculoskeletal TB [1,2]. However, lumbosacral segment TB is rare and only accounts for $2 \%-4 \%$ cases of

\footnotetext{
Received Mar 7, 2016; Revised Jul 15, 2016; Accepted Jul 16, 2016

Corresponding author: Xu Lan

Department of Spine Surgery, Lanzhou General Hospital, Lanzhou Command of Chinese PLA, 730050 Lanzhou, Gansu, China

Tel: +86-0931-8994323, Fax: +86-0931-8994006, E-mail: 444124272@qq.com

*Both authors contributed equally to this work.
} 
spinal TB $[3,4]$. TB spondylitis of the lumbosacral region has rarely been documented in the literature. The treatment of lumbosacral segment TB is difficult, and each patient needs individual consideration $[5,6]$. To date, there is a lack of consensus regarding the optimal therapeutic modality for treatment of lumbosacral segment TB.

Antituberculous treatment remains the cornerstone of therapy for spinal TB [7]. However, surgery may be required in selected cases, such as those with large abscesses, severe kyphosis, progressive neurological deficit, or lack of response to medical treatment. Various surgical approaches have been suggested for treating spinal TB, including debridement with anterior spinal fusion, anterior spinal fusion with posterior spinal fusion, posterior spinal fusion alone, and posterior spinal fusion followed by anterior spinal fusion $[8,9]$. Despite this, there was still contro- versy regarding the optimal surgical approach for treating lumbosacral segment TB. Anterior radical resection and bone grafting is a very important technique because it allows direct access to the affected vertebral segments and allows the surgeon to perform radical surgical debridement of the infected tissues $[10,11]$. Anterior approaches for lumbosacral TB include the extraperitoneal and transperitoneal approaches. The effective surgical approaches and methods for lumbosacral TB are controversial. Because the anatomy of lumbosacral segment is unique, the anatomy of the structures surrounding the lumbosacral segment is complicated and performing procedures in this area is difficult. One-stage anterolateral extraperitoneal approach for radical debridement, bone grafting, and internal instrument fixation for treating lumbosacral segment TB is rare in literature. Hence, the purpose of

Table 1. Patients' demogra with a larger number of patients are warranted

\begin{tabular}{|c|c|c|c|c|c|c|c|c|}
\hline Case No. & Age (yr) & Sex & Level & $\begin{array}{l}\text { Duration of } \\
\text { symptoms } \\
\text { (mo) }\end{array}$ & $\begin{array}{l}\text { Follow-up } \\
\qquad(\mathrm{mo})\end{array}$ & $\begin{array}{l}\text { Operation } \\
\text { time (min) }\end{array}$ & $\begin{array}{l}\text { Blood loss } \\
\text { (mL) }\end{array}$ & Complication \\
\hline 1 & 22 & Male & L5-S1 & 10 & 9 & 130 & 500 & None \\
\hline 2 & 60 & Femlae & L5-S1 & 12 & 12 & 160 & 400 & None \\
\hline 3 & 58 & Male & L4-S1 & 8 & 24 & 160 & 450 & None \\
\hline 4 & 18 & Male & L5-S1 & 8 & 12 & 210 & 800 & None \\
\hline 5 & 45 & Female & L5-S1 & 15 & 24 & 200 & 300 & None \\
\hline 6 & 28 & Male & L5-S1 & 9 & 24 & 140 & 400 & None \\
\hline 7 & 55 & Male & L5-S1 & 10 & 10 & 150 & 550 & None \\
\hline 8 & 62 & Male & L5-S1 & 18 & 18 & 130 & 200 & None \\
\hline 9 & 48 & Female & L5-S1 & 12 & 12 & 180 & 1,000 & None \\
\hline 10 & 35 & Male & L5-S1 & 6 & 12 & 160 & 500 & None \\
\hline 11 & 52 & Male & L5-S1 & 12 & 20 & 150 & 900 & None \\
\hline 12 & 38 & Male & L5-S1 & 8 & 9 & 120 & 300 & None \\
\hline 13 & 46 & Male & L4-S1 & 6 & 24 & 160 & 400 & None \\
\hline 14 & 52 & Female & L5-S1 & 18 & 20 & 140 & 700 & None \\
\hline 15 & 56 & Male & L5-S1 & 9 & 48 & 150 & 600 & None \\
\hline 16 & 26 & Male & L5-S1 & 9 & 18 & 160 & 350 & None \\
\hline 17 & 32 & Male & L5-S1 & 6 & 12 & 260 & 1,400 & $Y_{e s}{ }^{a)}$ \\
\hline 18 & 35 & Male & L5-S1 & 10 & 12 & 150 & 600 & None \\
\hline 19 & 57 & Female & L5-S1 & 8 & 9 & 180 & 400 & None \\
\hline 20 & 51 & Female & L5-S1 & 7 & 24 & 140 & 300 & None \\
\hline 21 & 70 & Male & L5-S1 & 10 & 12 & 130 & 500 & None \\
\hline Mean $\pm S D$ & $45.0 \pm 14.5$ & - & - & $10.0 \pm 3.5$ & $17.4 \pm 9.1$ & $160.0 \pm 32.2$ & $550.0 \pm 282.4$ & - \\
\hline
\end{tabular}

SD, standard deviation.

a)Laceration of common iliac vein. 
this clinical study was to evaluate the clinical efficacy and feasibility of the anterolateral extraperitoneal approach for treating lumbosacral TB.

\section{Materials and Methods}

\section{Clinical data}

Between May 2008 and May 2013, 21 patients with lumbosacral segment TB were treated in our hospital by the same surgeon. The subjects consisted of 15 men and six women with a mean age $45.0 \pm 14.5$ years (range, $18-70$ years). The duration of clinical symptoms ranged from 6 to 18 months (average, $10.0 \pm 3.5$ months) (Table 1).

All patients had different degrees of low-back pain as the primary symptom. Fourteen patients presented with clinical signs of TB infection, including low-grade fever, night sweats, loss of weight, and asthenia. Four patients had concurrent neurological disabilities. The American Spinal Injury Association (ASIA) impairment scale was used to evaluate neurological dysfunction. One patient grade-C dysfunction and three had grade-D dysfunction.

All patients underwent plain radiography, computed tomography (CT), magnetic resonance imaging along with measurements of the erythrocyte sedimentation rate (ESR) and C-reactive protein (CRP). Imaging revealed osteolysis in the L4-S1 region (Fig. 1). The lumbosacral angle ranged from $14^{\circ}$ to $31^{\circ}$ (average, $20.0^{\circ} \pm 4.8^{\circ}$ ). ESR ranged from 22 to $73 \mathrm{~mm} / \mathrm{hr}$ (average, $38.1 \pm 12.5 \mathrm{~mm} / \mathrm{hr}$ ) and CRP ranged from 0.5 to $13.7 \mathrm{mg} / \mathrm{dL}$ (average, $6.2 \pm 4.2$ $\mathrm{mg} / \mathrm{dL}$ ). The visual analogue score (VAS) and Oswestry disability index (ODI) were used to evaluate low-back pain and spinal disability. The VAS score ranged from 2 to 6 (average, 4.6 \pm 1.1 ) and ODI score ranged from $20 \%$ to $64 \%$ (average, $50.2 \% \pm 11.9 \%$ ) (Table 2).

\section{Preoperative preparation}

All patients were given antituberculous drugs, including isoniazid (300 $\mathrm{mg} /$ day), rifampicin ( $450 \mathrm{mg} /$ day), pyrazinamide (1,500 mg/day), and ethambutol (750 mg/day) for 3-4 weeks before surgery. ESR and CRP levels were measured to monitor antituberculous efficacy. In addition, a comprehensive assessment of the heart and pulmonary function was performed, and hypoalbuminemia was treated before operation.

\section{Operative procedure}

The patients were placed in the lateral position after general endotracheal anesthesia. All patients underwent surgery through an anterolateral extraperitoneal approach to the spine. Generally, the incision was made from the side where the vertebral bodies were severely damaged or the side of obvious abscess formation. Peritoneum that was protected by wet gauze was pushed forward in front of the iliacus muscle. The TB foci in L4-S1 and the vessels around lumbosacral vertebral bodies were exposed. Under $\mathrm{C}$-arm fluoroscopy guidance, the damaged vertebrae were located. Iliolumbar vein and ascending vein were ligated to avoid tearing of iliac veins through excessive retraction. Subperiosteal dissection was executed to protect the com-
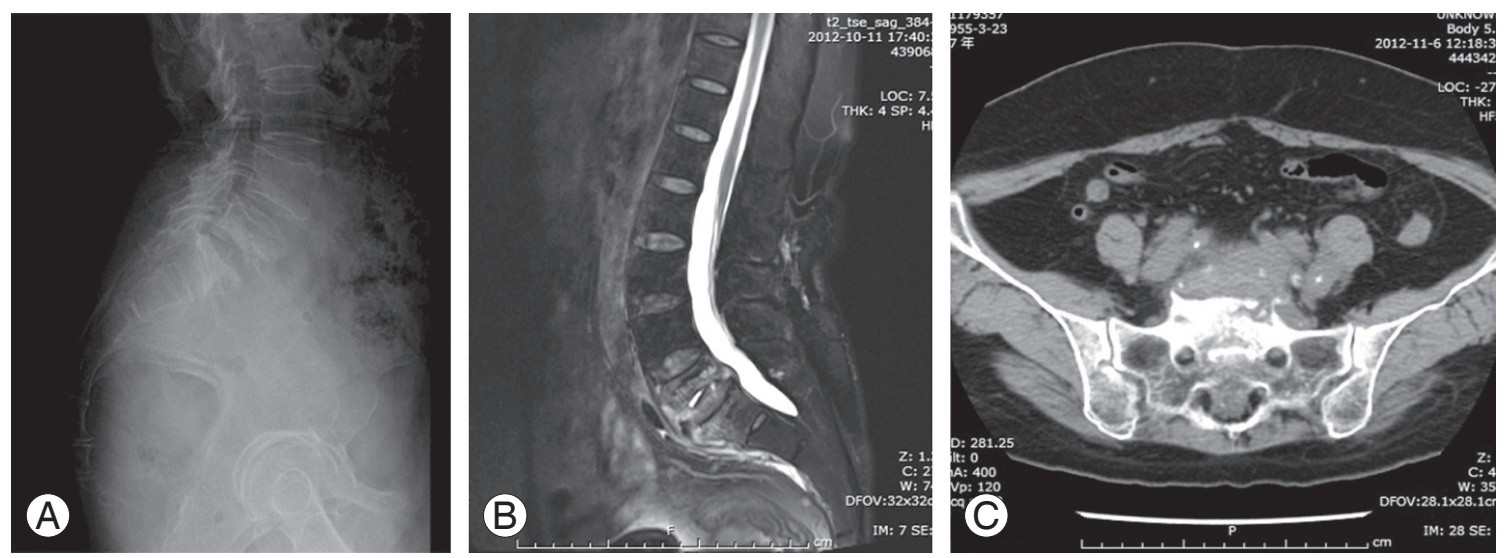

Fig. 1. (A) Lateral plain radiographs of the lumbosacral region shows destruction of $L 5, S 1$ vertebral bodies. (B) Magnetic resonance imaging shows tuberculosis at L5-S1 segment and paravertebral abscess formation. (C) Computed tomography shows the osteolysis in the L5 and S1 vertebrae. 


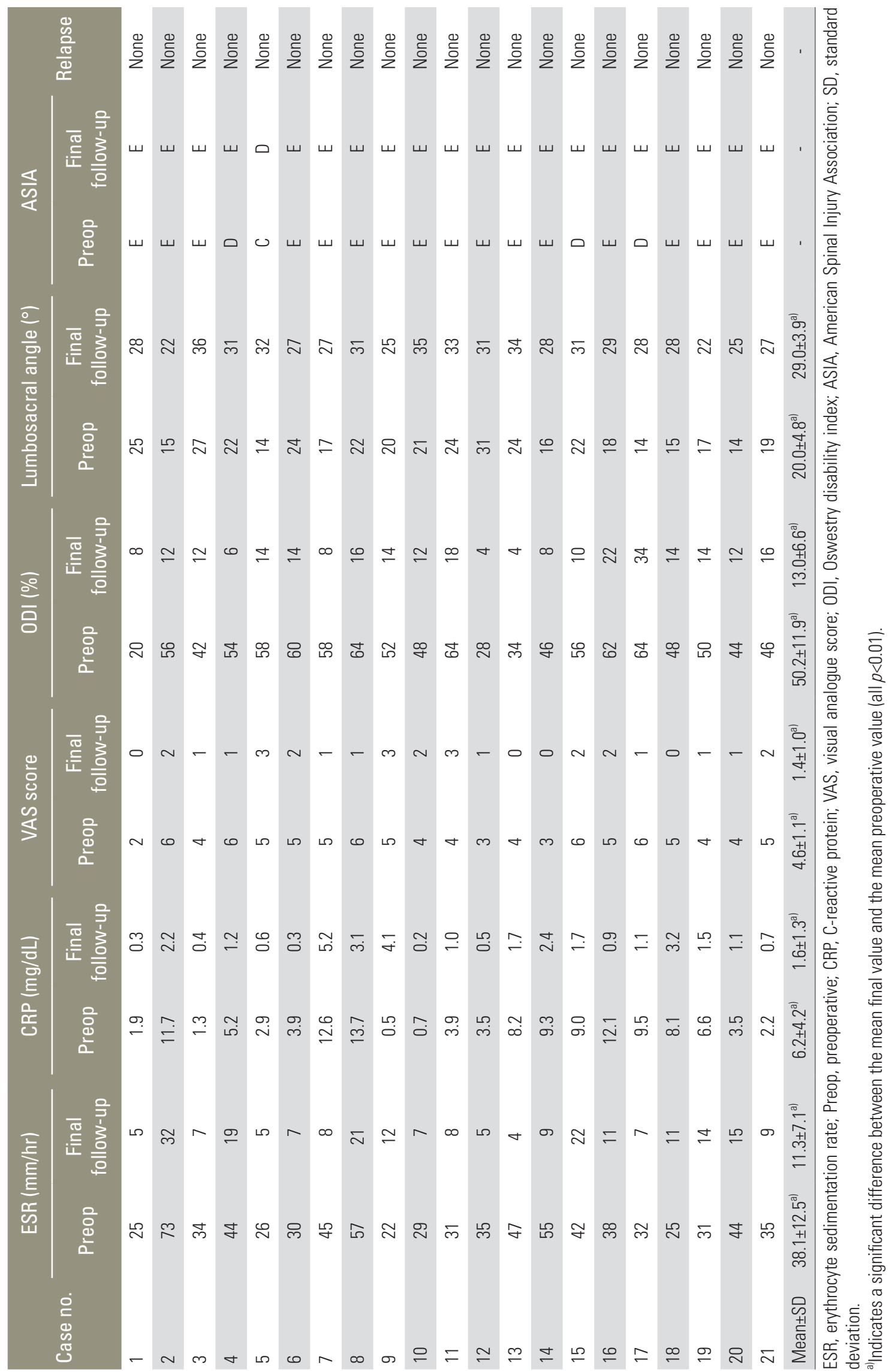


mon iliac vein and paravertebral soft tissue. After exposure, curettes and rongeurs were used to remove lesions, including sequestrae, abscesses, caseous material, and granulation tissue until the normal bleeding bone was exposed. The spinal canal was decompressed if there was any space-occupying lesion. The abscesses were drained and necrotic tissue was curetted as thoroughly as possible. The operative field was then washed with copious amounts of saline until the washing liquid was clear. A part of iliac crest was resected for autologous bone strut grafting and to facilitate the installation of internal fixation. The screws were inserted through the lateral aspects of the vertebral bodies to the bony cortex of the other side. Correction of spinal deformity was accomplished by installing the internal instrument with appropriate distraction. The spinal defect was then measured and the gap was repaired with a suitable length of autologous bone graft harvested from the ilium. The rods were applied lateral to the vertebral bodies and the bone graft was immobilized with appropriate compression. After careful hemostasis, $500 \mathrm{mg}$ of isoniazid and 1,000 $\mathrm{mg}$ of streptomycin were placed in the operative area, and a drainage tube was inserted before suturing the incision.

\section{Postoperative treatment}

The drainage tube was left in place for 48-96 hours. The drainage tube was removed when the volume of drainage was $<50 \mathrm{~mL}$ over a 24-hour period. Passive movements and functional limb exercises were carried out as early as possible. Conventional antituberculous pharmacotherapy was administered with isoniazid (300 $\mathrm{mg} /$ day), rifampicin (450 mg/day), pyrazinamide (1,500 $\mathrm{mg} /$ day $)$, and ethambutol (750 mg/day) for 3 months after surgery, which was followed by isoniazid ( $300 \mathrm{mg} /$ day), rifampicin (450 mg/day), and ethambutol (750 mg/day) for another 9-15 months. Patients were instructed to undergo routine blood examination to monitor ESR and CRP levels as well as liver and kidney functions. Patients were advised bed rest for a minimum of 6 weeks. Active and actively assisted exercises were advised. Complete extension exercises of abdominal, sacrospinal, iliopsoas, gluteus maximus, gluteus minimus, hamstring, quadriceps muscles as well as specific and resistive exercises of oblique abdominal muscles were prescribed. Exercises for augmenting respiratory capacity were also given. Mobilization was started after 6 weeks under external brace support. All patients wore braces for 6 months.

\section{Follow-up assessment}

We surveyed the wound healing rate and the recurrence rate of spinal TB. VAS and ODI scores were used to assess pain and spinal disability before and after surgery. ESR and CRP tests were used to monitor the antituberculous therapeutic efficacy and therapeutic reaction. Lumbar plain radiographs and/or CT examinations were performed to assess the lumbosacral angle correction and bony fusion. Achievement of union was determined by the formation of continuous bony trabeculae between the graft and host bone [12]. Neurological function was assessed using the ASIA classification before and after surgery. Evaluations of VAS, ODI, ESR, CRP, plain radiographs, and ASIA score were performed at 3, 6, 9, and 12 months after surgery. CT scans were performed at 6,9 or 12 months after surgery.

\section{Statistical analysis}

A paired $t$-test was used to analyze ESR, CRP, VAS score, ODI score, and lumbosacral angle between preoperative and final follow-up values. $p<0.05$ indicated statistical significance. We used SPSS ver. 17.0 for Windows (SPSS Inc., Chicago, IL, USA) for all statistical calculations.

\section{Results}

The mean duration of surgery was $160.0 \pm 32.2$ minutes (range, 120-260 minutes) and the mean volume of blood loss was 550.0 $\pm 282.4 \mathrm{~mL}$ (range, 200-1,400 mL) (Table 1). One patient had the complication of iliac vein tear during surgery. The rest of patients had no complications, neural disabilities, or ureteral injuries. All males were free of complications of erectile dysfunction and retrograde ejaculation. All patients' wounds healed well without chronic infection or sinus formation, and all symptoms of lowback pain were relieved after surgery. Patients complied with the follow-up survey for 9-48 months. All cases were cured, and no relapses were documented during the follow-up period. All patients obtained bony fusion within 6-12 months after surgery (average, 7.6 \pm 2.0 months) (Fig. 2 ). Four patients had evidence of neurological impairment before the operation. At the final follow-up assessment, the neurological status of the four patients with preopera- 

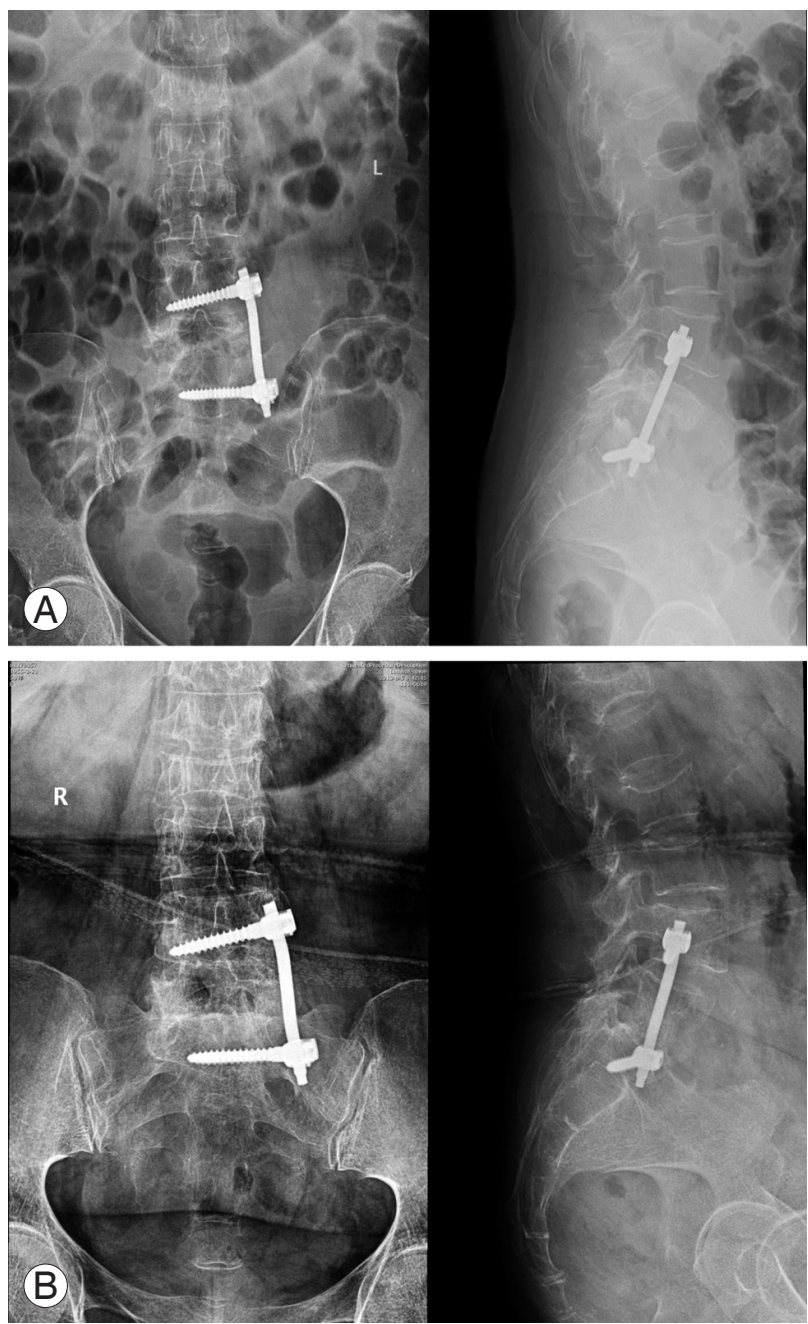

Fig. 2. (A) Anteroposterior and lateral plain radiographs after surgery reveals that the fixation is in a good position. (B) Anteroposterior and lateral plain radiographs reveal bony union 9 months after surgery. phics and operation information

tive neurological deficit was: one with grade $\mathrm{C}$ recovered to grade $\mathrm{D}$, three with grade $\mathrm{D}$ recovered to grade $\mathrm{E}$.

The average ESR was $38.1 \pm 12.5 \mathrm{~mm} / \mathrm{hr}$ preoperatively, which dropped to $11.3 \pm 7.1 \mathrm{~mm} / \mathrm{hr}(p<0.05)$. The average CRP was $6.2 \pm 4.2 \mathrm{mg} / \mathrm{dL}$ preoperatively, which declined to $1.6 \pm 1.3 \mathrm{mg} / \mathrm{dL}(p<0.05)$. The VAS and ODI scores before surgery were $4.6 \pm 1.1$ and $50.2 \% \pm 11.9 \%$, respectively, which decreased to $1.4 \pm 1.0$ and $13.0 \% \pm 6.6 \%(p<0.05)$. The average lumbosacral angle was $20.0^{\circ} \pm 4.8^{\circ}$ preoperatively, and this increased to $29.0^{\circ} \pm 3.9^{\circ}(p<0.05)$ (Table 2).

\section{Discussion}

Although spinal TB is a medical disease and antituberculous pharmacotherapy has a main role in the recovery of patients, surgical procedures need to be performed when patients develop large soft-tissue abscesses, cord or radicular compression, highly destructive lesions producing spinal bone instability, or severe pain without relief under medical treatment. Surgical treatment of spinal TB can result in spinal stabilization and early mobilization. Surgery can also facilitate the success of chemotherapy, because the abscess cavity provides an avascular environment that protects TB bacilli from systemic antibiotics, and local sclerotic bone prevents permeation of antibiotics [13-15]. Thus, we propose that surgical intervention should be performed for treating lumbosacral TB with abscess formation, severe vertebral destruction, neural dysfunction, or unsuccessful conservative treatment. All the necrotic tissue should be debrided, the abscess cavity should be destroyed, and the sclerotic bone should be removed during surgery. Consequently, the risk of spinal TB relapse will be low, and the effect of antituberculous pharmacotherapy will be enhanced. In the end, all the patients in our study who underwent radical debridement were cured and no relapse has been reported.

The conventional surgical approach to lumbar and sacral vertebral TB is posterior instrumented fixation and deformity correction followed by anterior debridement and fusion with bone grafting. However, spinal TB mainly affects the anterior and middle vertebral columns, leading to vertebral bone defects, collapse, compression, and kyphosis. Anterior-column and middle-column damage accounts for $98 \%$ cases of spinal TB, while the posteriorcolumn structure usually remains intact [16]. Biedemann [17] found that in an intact vertebral column, $80 \%$ load is transmitted through the anterior column and $20 \%$ through the posterior column. Therefore, we suggest that the anterior approach is the preferred solution for lumbosacral TB.

Because anterior debridement and arthrodesis for treating spinal TB was reported by Hodgson and Stock [18], several surgeons have advocated this procedure and emphasized its advantages as reaching the focal point of the disease directly, enabling effective debridement of the lesion, and decompression of the spinal cord. However, radical debridement can produce spinal instability. Therefore, anterior strut grafting and instrumentation should be preferred in cases with spinal instability [9]. The anterolateral approach is our surgeons' preferred method as well, although different approaches have their own advantages and disadvantages [19-24]. In our study, 
all patients underwent one-stage anterolateral extraperitoneal approach for radical debridement, lateral autologous strut grafting, and single screw-rod instrumentation for lumbosacral TB. The anterolateral approach can achieve direct visualization of the TB focus and facilitate direct debridement and bone grafting. In addition, the lumbosacral debridement using this approach is more complete. Although the recurrence rate for skeletal TB is approximately $2 \%$ [7], there was no relapse in the light of complete debridement and multidrug therapy in our study. The extraperitoneal approach can prevent the development of intra-abdominal TB infections and decrease the impact on abdominal organs. In this study, there were several patients with temporary abdominal distension and constipation after surgery. All postoperative complications were relieved after symptomatic treatment. With the anterolateral extraperitoneal approach for lumbosacral $\mathrm{TB}$, the surgeon can achieve debridement, bone grafting, and internal fixation at the same time. There was no need to change the patients' position during surgery, shortening the operative duration and decreasing blood loss. Anterolateral instrument and strut grafting can preferably correct the lumbosacral deformity and at the same time immobilize the bone autograft. The average lumbosacral angle was $\left(20.0^{\circ} \pm 4.8^{\circ}\right)$ before surgery, and this improved to $\left(29.0^{\circ} \pm 3.9^{\circ}\right)$ at follow-up. Studies have shown a direct correlation between a decrease in normal lordosis of the lower lumbar and lumbosacral spine with the severity of low-back pain. In this paper, the low-back pain was ameliorated immediately after the patients' lumbosacral angle was increased to its optimal state; all patients' low-back pain improved after correction of the lumbosacral angles that would alleviate shear stress in the juncturae lumbosacralis. There was no migration of the bone autograft after anterolateral internal fixation, and there was no looseness or fracture of the internal instruments during the followup period. We conclude, therefore, that one-stage anterolateral debridement, bone grafting, and internal fixation for treating lumbosacral TB is safe and feasible.

Of note, all postoperative patients need to stay in bed for a minimum of 6 weeks. Following that, patients need to be on an external brace for 6 months. Early ambulation is not conducive to spinal stability. However, there are many complications of prolonged bed rest, such as hypostatic pneumonia and thrombus formation in the lowerextremity veins. All patients, wearing an external brace, should stare exercises 6 weeks after surgery. The potential benefits of wearing the brace include limiting spinal range of motion, correcting posture and deformity, preventing gross trunk motion, decreasing force exerted by trunk muscles, providing soft-tissue massage and heat, and improving spinal proprioception [25]. Moreover, bracing is helpful for treating low-back pain. Calmels et al. [26] proposed bracing for the short-term management of subacute low-back pain. Although there is no evidence regarding the effect of lumbar bracing on the rate of lumbar spinal fusion or functional outcome, several authors have advocated the use of brace therapy following lumbar fusion surgery [27].

Our experience with the anterolateral extraperitoneal approach for lumbosacral TB is as follows: (1) patients are placed in the lateral position and the lower extremity of the operational side is flexed to avoid stretch injury to the lumbar nerve root. (2) The iliolumbar vein and ascending vein are ligated before debridement to avoid iliac vein tears and massive hemorrhage. (3) Surgeons should avoid excessive pulling of soft tissue around the anterolateral vertebral bodies; otherwise, this will cause iliac vein or sympathetic trunk injury. (4) The iliac wing needs to be resected to facilitate the installation of internal instruments before embedding the vertebral screws.

The anterolateral extraperitoneal approach for lumbosacral TB has many advantages, but this approach also has a few limitations that should be considered. First, it is much more difficult to expose the lumbosacral TB focus in an overweight or obese patient. Second, the sacral screw is difficult to adequately fix to the $S 1$ vertebral body when the $S 1$ is severely damaged. When the $S 1$ is severely destroyed, a simultaneous posterior approach of internal fixation may be needed with anterior debridement. Furthermore, the anterolateral approach is not appropriate for every surgical facility. The surgeons' experience of the anterolateral approach is vital for the success of the surgery. Otherwise, there will have difficulties and risks during surgery if the surgeon is not familiar with the anatomic structure of the lumbosacral segment. Further studies

\section{Conclusions}

Lumbosacral TB is not the common form of TB spondylitis. However, it also can cause severe clinical consequences and become a serious social and economic burden; hence, early diagnosis and treatment are vital. Our study showed that surgical treatment of lumbosacral TB using 
one-stage anterolateral extraperitoneal debridement, bone grafting, and internal fixation achieved satisfactory clinical outcomes and achieved good results in spinal stability reconstruction. On the basis of the reported literature and the positive therapeutic effect in our patient population, we propose that lumbosacral TB can be efficiently treated with one-stage anterolateral extraperitoneal debridement, bone grafting and internal instrument fixation.

\section{Conflict of Interest}

No potential conflict of interest relevant to this article was reported.

\section{References}

1. Gautam MP, Karki P, Rijal S, Singh R. Pott's spine and paraplegia. JNMA J Nepal Med Assoc 2005;44:10615.

2. Talbot JC, Bismil Q, Saralaya D, Newton DA, Frizzel RM, Shaw DL. Musculoskeletal tuberculosis in Bradford: a 6-year review. Ann R Coll Surg Engl 2007;89: 405-9.

3. Rajasekaran S, Shanmugasundaram TK, Prabhakar R, Dheenadhayalan J, Shetty AP, Shetty DK. Tuberculous lesions of the lumbosacral region: a 15-year follow-up of patients treated by ambulant chemotherapy. Spine (Phila Pa 1976) 1998;23:1163-7.

4. Sakho Y, Badiane SB, N'Dao AK, N'Diaye A, Gueye M, N'Diaye IP. Pott's disease in Senegal. Eur J Orthop Surg Traumatol 2003;13:13-20.

5. Pun WK, Chow SP, Luk KD, Cheng CL, Hsu LC, Leong JC. Tuberculosis of the lumbosacral junction: long-term follow-up of 26 cases. J Bone Joint Surg Br 1990;72:675-8.

6. Shah SP, Kulkarni AG. S1 tuberculosis treated with segmental lumbopelvic fixation: a case report. Spine (Phila Pa 1976) 2012;37:E866-9.

7. Tuli SM. Tuberculosis of the spine: a historical review. Clin Orthop Relat Res 2007;460:29-38.

8. Moon MS, Moon YW, Moon JL, Kim SS, Sun DH. Conservative treatment of tuberculosis of the lumbar and lumbosacral spine. Clin Orthop Relat Res 2002;(398):40-9.

9. Bezer M, Kucukdurmaz F, Aydin N, Kocaoglu B, Guven O. Tuberculous spondylitis of the lumbosacral region: long-term follow-up of patients treated by chemotherapy, transpedicular drainage, posterior instrumentation, and fusion. J Spinal Disord Tech 2005;18:425-9.

10. Aryan HE, Lu DC, Acosta FL Jr, Ames CP. Standalone anterior lumbar discectomy and fusion with plate: initial experience. Surg Neurol 2007;68:7-13.

11. Zhang T, He X, Li H, Xu S. Treatment of lumbosacral spinal tuberculosis by one-stage anterior debridement and fusion combined with dual screw-rod anterior instrumentation underneath the iliac vessel. BMC Musculoskelet Disord 2016;17:49.

12. Suk SI, Lee CK, Kim WJ, Lee JH, Cho KJ, Kim HG. Adding posterior lumbar interbody fusion to pedicle screw fixation and posterolateral fusion after decompression in spondylolytic spondylolisthesis. Spine (Phila Pa 1976) 1997;22:210-9.

13. Ge Z, Wang Z, Wei M. Measurement of the concentration of three antituberculosis drugs in the focus of spinal tuberculosis. Eur Spine J 2008;17:1482-7.

14. Liu $P, Z$ hu $Q$, Jiang J. Distribution of three antituberculous drugs and their metabolites in different parts of pathological vertebrae with spinal tuberculosis. Spine (Phila Pa 1976) 2011;36:E1290-5.

15. Jin W, Wang Q, Wang Z, Geng G. Complete debridement for treatment of thoracolumbar spinal tuberculosis: a clinical curative effect observation. Spine J 2014;14:964-70.

16. Jin W, Wang Z. Clinical evaluation of the stability of single-segment short pedicle screw fixation for the reconstruction of lumbar and sacral tuberculosis lesions. Arch Orthop Trauma Surg 2012;132:1429-35.

17. Biedemann L. Biomecanica of pedicle fixation as related to implant design. In: 1st American-European meeting on pedicle fixation of the spine and other advanced techniques; 1994 Jul 1-2, Munich, Germany. Munich: Springer; 1994. p.115.

18. Hodgson AR, Stock FE. Anterior spine fusion for the treatment of tuberculosis of the spine: the operative findings and results of treatment in the first one hundred cases. J Bone Joint Surg Am 1960;42:295-310.

19. Song JF, Jing ZZ, Chen B, Ai ZS, Hu W. One-stage anterolateral surgical treatment for lumbosacral segment tuberculosis. Int Orthop 2012;36:339-44.

20. $\mathrm{He} \mathrm{Q}, \mathrm{Xu}$ J. Comparison between the antero-posterior and anterior approaches for treating L5-S1 vertebral tuberculosis. Int Orthop 2012;36:345-51.

21. Wang Z, Wu Q, Geng G. Anterior debridement and 
bone grafting with posterior single-segment internal fixation for the treatment of mono-segmental spinal tuberculosis. Injury 2013;44:253-7.

22. Mak KC, Cheung KM. Surgical treatment of acute TB spondylitis: indications and outcomes. Eur Spine J 2013;22 Suppl 4:603-11.

23. Wang Z, Yuan H, Geng G, Shi J, Jin W. Posterior mono-segmental fixation, combined with anterior debridement and strut graft, for treatment of the mono-segmental lumbar spine tuberculosis. Int Orthop 2012;36:325-9.

24. Lan X, Xu J, Liu X, Ge B. Debridement and allograft with internal fixation via combined anterior and posterior approach for treatment of lumbosacral tuber- culosis. Zhongguo Xiu Fu Chong Jian Wai Ke Za Zhi 2011;25:1176-9.

25. Dailey AT, Ghogawala Z, Choudhri TF, et al. Guideline update for the performance of fusion procedures for degenerative disease of the lumbar spine. Part 14: brace therapy as an adjunct to or substitute for lumbar fusion. J Neurosurg Spine 2014;21:91-101.

26. Calmels P, Queneau P, Hamonet C, et al. Effectiveness of a lumbar belt in subacute low back pain: an open, multicentric, and randomized clinical study. Spine (Phila Pa 1976) 2009;34:215-20.

27. Connolly PJ, Grob D. Bracing of patients after fusion for degenerative problems of the lumbar spine: yes or no? Spine (Phila Pa 1976) 1998;23:1426-8. 